\title{
Physiological Need for Calcium, Iron, and Folic Acid for Women of Various Subpopulations During Pregnancy and Beyond
}

\author{
LaVerne L. Brown, PhD, ${ }^{1}$ Barbara E. Cohen, PhD, MPH, ${ }^{1}$ Emmeline Edwards, PhD, ${ }^{2}$ \\ Courtney E. Gustin, DrPH, CNM, RN, ${ }^{3}$ and Zara Noreen, $\mathrm{BA}^{4}$
}

\begin{abstract}
Women tend to supplement their diets with multivitamin/mineral (MVM) supplements more often than men, and reports indicate that more than $90 \%$ of pregnant women in the United States supplement their diets with prenatal MVMs. Given the widespread use of MVMs among women and given the increasing efforts to unveil the importance of phenotype-specific health determinants, it seems imperative to review what is known about variations in nutrient physiology among women from different ethnic and racial groups and at different reproductive stages of life. In this study, we embark on an assessment of the scientific evidence and knowledge gaps that impact the precise determination of nutrient levels (specifically calcium, iron, and folic acid) that confer benefits to various subpopulations of women in the United States.
\end{abstract}

Keywords: calcium, folic acid, iron, pregnancy

D URING PREGNANCY, WOMEN are advised to consume higher levels of calcium, iron, and folic acid. As research continues to unveil the importance of phenotypespecific health determinants, it is critical to review what is known about nutrient physiology and how it may vary among women from different ethnic and racial groups and at different reproductive stages of life. This article presents the current understanding of calcium, iron, and folic acid physiology; related health consequences for women; and research gaps that impact the precise determination of nutrient levels and intake practices that confer health benefits to women.

\section{Calcium}

Calcium, an essential element available only through dietary sources, is responsible for maintenance of the electric gradient across membranes, bone formation, and enzyme and hormone functioning. Stored largely in bone (99\%), calcium is essential for muscle contraction, bone and tooth development, blood clotting, nerve impulse transmission, heart rhythm regulation, and fluid balance within cells. ${ }^{1,2}$

Calcium balance is a function of dietary intake, intestinal absorption, renal excretion, and bone remodeling. The three latter mechanisms are regulated by the parathyroid hormone (PTH), 1,25-dihydroxyvitamin D [1,25(OH)2D], and ionized calcium, along with receptors in the intestine, kidney, and bone that correspond to these hormones. ${ }^{3}$ Hypercalcemia, frequently caused by primary hyperparathyroidism and malignancy, is associated with depression, lethargy, coma, muscle weakness, and constipation. ${ }^{1}$ Hypocalcemia-normally caused by medication, medical treatment, or disease processes - can result in convulsions, paresthesia, cramps, and tetany. ${ }^{1}$ Dietary deficiency can cause muscle cramping, dry skin, and brittle

\footnotetext{
${ }^{1}$ Office of Dietary Supplements, National Institutes of Health, Bethesda, Maryland, USA.

${ }^{2}$ National Center for Complementary and Integrative Health, Division of Extramural Research, National Institutes of Health, Bethesda, Maryland, USA.

${ }^{3}$ Office of Women's Health, U.S. Department of Health and Human Services, Rockville, Maryland, USA.

${ }^{4}$ Office of Research on Women's Health, National Institutes of Health, Bethesda, Maryland, USA.

(c) LaVerne L. Brown et al. 2021; Published by Mary Ann Liebert, Inc. This Open Access article is distributed under the terms of the Creative Commons Attribution Noncommercial License (http://creativecommons.org/licenses/by-nc/4.0/) which permits any noncommercial use, distribution, and reproduction in any medium, provided the original author(s) and the source are cited.

Correction added on February 19, 2021 after first online publication of November 7, 2020: The article reflects Open Access, with copyright transferring to the author(s), and a Creative Commons Attribution Noncommercial License (CC-BY-NC) added (http:// creativecommons.org/licenses/by-nc/4.0/).
} 
nails. It can also lead to osteoporosis and its associated effects, including bone deterioration and increased risk of fractures. ${ }^{2}$

Calcium requirements vary in women, depending on their reproductive stage. ${ }^{4,5}$ Calcium bone stores fluctuate throughout a woman's life, with absorption from the diet gradually declining in adulthood. ${ }^{5}$

Bone growth occurs slowly during early childhood, increases quickly during puberty, and peaks between 25 and 30 years of age. Inadequate calcium intake can cause several problems in growing children and adolescents, including stunted growth and reduced peak bone density. ${ }^{6,7}$

During pregnancy, the efficiency of intestinal calcium absorption doubles, whereas during lactation, the maternal skeleton is resorbed to provide calcium for milk. Calcium intake remains especially critical during pregnancy and lactation because of the potential adverse effect on maternal and fetal bone health if maternal calcium stores are depleted. Since both the fetus and breastfed newborn are dependent on maternal sources for their total calcium, adequate maternal calcium intake can also affect fetal bone health positively. ${ }^{8}$

Inadequate calcium during pregnancy also impacts the development of pregnancy-related hypertensive disorders. Low calcium intake may stimulate PTH secretion, increasing intracellular calcium and smooth muscle contractions. Additionally, it can cause the release of renin from the kidney, leading to vasoconstriction and retention of sodium and fluid. These changes can lead to the development of pregnancyinduced hypertension (PIH) and preeclampsia. ${ }^{9}$

At menopause, a decline in intestinal calcium absorption and/ or an increase in urinary calcium excretion may occur, leading to deterioration in calcium balance. ${ }^{10,11}$ Recent data (2015) from clinical trials suggest that the effect of supplemental calcium is minimal or nonexistent for fracture prevention. ${ }^{12}$

Although numerous health consequences of calcium insufficiency and surplus are found in all populations, ${ }^{13-15}$ data suggest that calcium metabolism and utilization differ by ethnicity. ${ }^{16,17}$ Black adolescents absorb and retain calcium more efficiently than Whites, and bone turnover is greater in Blacks, favoring net bone accretion. These differences may account for bone mass differences observed in adulthood. ${ }^{18}$ The incidence of osteoporosis and osteopenia is greater among nonHispanic White and Asian adult women than among nonHispanic Black and Hispanic women of all races. Peak bone mass, a predictor of fracture risk, is highest in Black women, who have the lowest incidence of fracture, lower in Hispanic and non-Hispanic White women, and lowest in Asian American women. However, some Asian subgroups with very low peak bone mass also have lower fracture rates. Ethnic variations in bone metabolism may be related to inherited genetic polymorphisms. Among ethnic groups, there are variations in acid metabolism, resulting in ethnic variations in sodium and calcium excretion rates; differences in bone turnover; and differences in intestinal calcium absorption. Such variations in metabolism may explain higher bone mineral density in African Americans. ${ }^{19}$

Given that adequate calcium intake during pregnancy and lactation is critical for the health of both mother and fetus and that most women do not consume the recommended quantity of calcium at any time in their lives, calcium supplementation is considered. ${ }^{9}$ Two meta-analyses indicate the potential positive impact of calcium supplementation on $\mathrm{PIH}$ and preeclampsia. The first study demonstrated that the additional intake of calcium during pregnancy is an effective measure to reduce the incidence of preeclampsia, especially in populations at high risk due to ethnicity, gender, age, high body-mass index, and low baseline calcium intake. ${ }^{20}$ The second study suggests that for women with low calcium intake, calcium supplementation greater than $1 \mathrm{~g}$ per day may reduce the risk of preeclampsia, preterm birth, maternal deaths, and serious morbidities, but not stillbirth or admission to neonatal high care. ${ }^{21}$ The limited data on low-dose calcium supplementation suggest a reduction in preeclampsia, hypertension, and admission to neonatal high care.

Because calcium intake tends to decrease with age, calcium supplementation is especially important in postmenopausal women. In addition, because of a decrease in estrogen production after menopause, women's bodies are less able to retain calcium from dietary sources. Consequently, calcium supplementation has been used for decades to prevent calcium depletion, maintain bone mass, and prevent and treat osteoporosis. ${ }^{22,23}$

\section{Iron}

Iron is an essential nutrient that assists with the transfer of oxygen from the lungs to bodily tissues. A diet deficient in iron can negatively impact muscle metabolism, cognitive development, and growth. Excess iron intake results in oxidative stress, which is associated with hemochromatosis, some cancers, premature aging, neurodegenerative diseases, and atherosclerosis. ${ }^{24}$

Sex differences with respect to physiologic requirements for iron intake are well established. Adult females require more iron than adult males because of the loss of iron in their blood during menses. On the other hand, preadolescent males show a higher prevalence of iron deficiency anemia than preadolescent females. Iron levels tend to increase in postmenopausal women and decrease in males of similar age and status. $^{25}$ The Food and Nutrition Board at the National Academies of Sciences, Engineering, and Medicine therefore recommends higher dietary allowances of iron for females aged 14-50 years than for their male counterparts. In addition, because the physiologic requirements of iron in females are further complicated by pregnancy and lactation, current recommendations support higher dietary allowances of iron in pregnant females and lower dietary allowances of iron in lactating females than in nonpregnant and nonlactating females. ${ }^{26}$

In fact, maternal physiologic iron requirements fluctuate throughout pregnancy. During the first trimester, iron requirements decrease because of the cessation of menses, and iron stores may increase. During the second trimester, physiologic iron requirements increase due to expansion of maternal blood volume and red cell mass. Linear increases in iron requirements continue through the third trimester as iron accumulates in the placenta to support the development of infant red blood cells.

In healthy pregnant women with adequate prepregnancy iron stores, the increased iron requirements are balanced by more efficient iron absorption from the diet, improved oxygen delivery to the tissues, and increased heart output. ${ }^{27}$ Assuming that $300 \mathrm{mg}$ of stored iron reserves are adequate in prepregnant females, then $\sim 50 \%$ of women in the United States achieve this status. ${ }^{27}$ On the other hand, as many as $35 \%$ of women in the United States have depleted or nearly depleted iron reserves and are considered iron deficient. ${ }^{27}$ To offset a potential imbalance in iron reserves during pregnancy and prevent maternal iron deficiency anemia, routine iron supplementation is widely practiced. 
The U.S. Preventive Services Task Force (USPSTF) has published a statement indicating that evidence is insufficient to warrant routine iron supplementation during pregnancy. ${ }^{28}$ Current published evidence on the benefits and risks of routine iron supplementation during pregnancy tends to focus more on infant outcomes and less on maternal health. With respect to maternal health, physiologic requirements for iron intake-and thus the benefits and risks of routine iron supplementation - are complicated by confounding factors, including ethnicity, diet, lifestyle, genetics, preexisting chronic diseases and conditions, and methods for assessing precise measures of iron status.

Iron status is commonly assessed as a measure of hemoglobin concentrations. However, interpretations of the outcomes of these measures are problematic. For example, iron deficiency as a measure of hemoglobin concentration is not always associated with anemic outcomes in all populations. On the other hand, measures of serum ferritin, a biomarker for iron stores, are more often preferred as a metric for low iron status. ${ }^{29}$ Interpretations of high ferritin concentrations also have proven to be problematic. Because ferritin acts as an acute phase reactant to infection and inflammation, it is difficult to discern whether high levels of serum ferritin are indicative of an iron overload status or inflammation. ${ }^{30}$ Furthermore, data from the Hemochromatosis and Iron Overload Screening Study of 20,080 nonpregnant and 1962 pregnant women suggest innate biological differences in serum ferritin levels (as well as transferrin saturation) across racial and ethnic groups and also suggest that these measures must be interpreted differently in persons of Asian or Pacific Island heritage than in Whites. ${ }^{31}$ Similarly, differences between the interpretation of maternal hematocrit levels in Black pregnant women and in White pregnant women have been reported. ${ }^{32,33}$

Improved assessments of maternal iron status are crucial for determining for which individuals and to what extent iron supplementation might improve maternal health outcomes. High ferritin levels (even after accounting for inflammatory status) have been associated with insulin resistance, type 2 diabetes, and hypertension. A recent meta-analysis suggests that consumption of dietary heme iron may exacerbate the onset of gestational diabetes mellitus (GDM) ${ }^{34,35}$ Less is understood about the role of iron supplements and the onset of GDM. While nonheme forms of iron found in iron supplements are much less readily absorbed than dietary heme iron, some evidence suggests that in iron-replete women, consumption of iron supplements may result in increased amounts of unabsorbed iron in the intestine, damage of pancreatic $\beta$ cells, increased insulin resistance, and subsequent development of diabetes. ${ }^{29,34}$

These outcomes are especially relevant given the alarming rates of maternal morbidity in the United States. Precise recommendations of iron intake within the context of ethnicity, life stage, and preexisting conditions may offer a potential opportunity to mitigate risk factors associated with maternal morbidity.

\section{Folic Acid}

Folate, or vitamin B9, is a water-soluble vitamin that the body utilizes to synthesize nucleic acids (DNA and RNA) and to metabolize amino acids for cell growth. Although it is found in many foods (dark leafy greens, peas and beans, and fruits), many countries fortify grain and cereal products with folic acid (synthetic folate).
One of the benefits of folate when taken during pregnancy is the prevention of fetal neural tube defects (NTDs). Folate and folic acid also have shown benefits or potential benefits in the treatment of cardiovascular disease ${ }^{36-38}$ and diabetes, ${ }^{39}$ cancer and antiviral therapies, ${ }^{40,41}$ longer term neurological development in infants and neurological status in adults, ${ }^{42}$ treatment of such chronic diseases as arthritis ${ }^{43}$ and sickle cell disease, ${ }^{44}$ reduction of the effects of radiation exposure, ${ }^{45}$ and improvement in neurocognitive function ${ }^{42}$ and as additives in various treatments and medications.

Although the benefits of folic acid supplementation in preventing NTDs are clear, some concerns are associated with increased folate and folic acid intake. Folic acid supplementation may mask an existing vitamin B12 deficiency. ${ }^{46}$ Additionally, some studies have linked excess folic acid intake with increased risk of the development and recurrence of certain types of cancers. ${ }^{47}$

No gender-specific differences in folate or folic acid metabolism or biological requirements for men and women have been reported, except to accommodate reproductive differences. The National Institutes of Health's Office of Dietary Supplements recommends identical folic acid intake requirements for men and women throughout the life span, increasing from $65 \mu \mathrm{g}$ of dietary folate equivalents (DFE) at time of birth and up to 6 months to $400 \mu \mathrm{g}$ DFE in adulthood, with increases for pregnancy and lactation. ${ }^{48}$

Although studies show that most Americans have an adequate dietary intake of folate, pregnant women, teens, and other groups may have insufficient levels of folate. ${ }^{49}$ The USPSTF recommends that women who are planning to or could become pregnant take a folic acid supplement of 0.4 to $0.8 \mathrm{mg}$ daily to prevent NTDs. For maximum effectiveness, the supplementation must begin in the month before pregnancy and continue to the second or third month. ${ }^{50}$

Because of the high rate of unplanned pregnancy and the potential serious effects of folate deficiency, the Food and Drug Administration has mandated the fortification of such U.S. grain products as pasta, rice, and cereals since January 1998 and of corn masa flour since April $2016 .^{51}$

Estimating racial and ethnic differences in both folate and folic acid synthesis and the effects of fortification and supplementation on outcomes has been difficult. Supplementation with folic acid had less of an effect on maternal and cord blood sample folates for African Americans. ${ }^{52}$ A study of women living in Georgia found that $\sim 25 \%$ of women were taking a folic acid supplement preconception, with non-Hispanic women twice as likely as Hispanic women to take folic acid supplements. ${ }^{53}$ In terms of differences in metabolism, among women taking folic acid supplements, the largest increase in folate levels was noted in White women, followed by Hispanic women, with Black women experiencing the lowest serum folate levels. ${ }^{54}$

Underlying conditions also affect folate levels and risk for NTDs. Hispanic women are more likely than other groups to have the MTHFR C677T gene variant, which can result in impaired folic acid processing. ${ }^{55}$ People with alcohol use disorder, inflammatory bowel disease, celiac disease, gastric surgeries, and gastritis may be at risk for impaired folic acid absorption. ${ }^{56}$ Ethnic and racial variations in the prevalence of these conditions affect the physiology of folic acid.

When identifying the success and cost-benefit analysis of folic acid food enrichment, it is important to take cultural foods into account. Many wheat and other cereal-based 
products have been approved for enrichment, but many other grain-based foods have not; corn flour was not required to be enriched until 2016, which is significant because corn flour is commonly used in traditional Hispanic foods. The rates of folic acid consumption for Hispanics have been consistently lower than for Caucasians. ${ }^{57}$

\section{Conclusions}

Despite the wealth of research on calcium, iron, and folate and their effects on women's health outcomes, most studies conducted in the United States either included only White women or, when mixed ethnic/racial groups were included, the studies lacked adequate representation of specific groups that would allow appropriate subgroup analyses. Studies of specific ethnic and racial groups tend to be small and are inconsistent across all populations. Large longitudinal studies with adequate representation across ethnic and racial groups, which allows cross-group and intragroup comparisons, are needed. Intragroup analyses should separate regional differences or countries of origin since different cultural habits impact dietary intake and thus risk for certain diseases.

Additionally, intake data, generally obtained through dietary and supplement adherence recall methods, may not adequately capture the variation in the amount of each nutrient and their bioavailability in various supplements, the differences between the bioavailability of nutrients in food and supplements, and variations in prescribed intake protocols (for high-risk vs. low-risk groups). Research using standardized supplement doses or more carefully controlled dietary monitoring would improve the reliability of the data.

Finally, methodologies used to study health outcomes need to be refined. For example, bone health studies vary in bone test sites used, reporting methods, and sample sizes. Studies are needed to better determine the etiology of diseases related to calcium, iron, and folic acid within different population subgroups and should include larger high-quality trials.

\section{Author Disclosure Statement}

No competing financial interests exist.

\section{Funding Information}

No funding was received for this article.

\section{References}

1. Stewart AF. Calcium metabolism without anguish. Understanding the body's homeostatic 'black box.' Postgrad Med 1985;77:238-294.

2. Pravina P, Sayaji D, Avinash M. Calcium and its role in human body. Int J Pharm Biol Sci 2013;4:659-668.

3. Peacock M. Calcium metabolism in health and disease. Clin J Am Soc Nephrol 2010;5(Supplement 1):S23-S30.

4. Fordyce A, Gouliouk V, Henken RI. Dietary nutrient intake affects calcium and magnesium metabolism. FASEB J 2011;25(S1):594.14.

5. Abrams SA. Calcium turnover and nutrition through the life cycle. Proc Nutr Soc 2001;60:283-289.

6. Ross CA, Manson JA, Abrams SA, et al. The 2011 report on dietary reference intakes for calcium and vitamin $\mathrm{D}$ from the Institute of Medicine: What clinicians need to know. J Clin Endocrinol Metab 2011;96:53-58.
7. Chevalley T, Rizzoli R, Hans D, Ferrari S, Bonjour $\mathrm{P}$. Interaction between calcium intake and menarcheal age on bone mass gain: An eight-year follow-up study from prepuberty to postmenarche. J Clin Endocrinol Metab 2014;90:44-51.

8. Kovacs CS. Maternal mineral and bone metabolism during pregnancy, lactation, and post-weaning recovery. Physiol Rev 2016;6:449-547.

9. Hacker AN, Fung EB, King JC. Role of calcium during pregnancy: Maternal and fetal needs. Nutr Rev 2012;70: 397-409.

10. Spangler M, Phillips BB, Ross MB, Moores KG. Calcium supplementation in postmenopausal women to reduce the risk of osteoporotic fractures. Am J Health Syst Pharm 2011;68:309-318.

11. Cumming RG, Nevitt MC. Calcium for prevention of osteoporotic fractures in postmenopausal women. J Bone Miner Res 1997;12:1321-1329.

12. Bolland MJ, Leung W, Tai V, Bastin S, Gamble GD, Grey A, Reid IR. Calcium intake and risk of fracture: Systematic review. BMJ 2015;351:h4580.

13. Belizán JM, Villar J, Repke J. The relationship between calcium intake and pregnancy-induced hypertension: Upto-date evidence. Am J Obstet Gynecol 1988;158:898-902.

14. Zemel MB. Calcium modulation of hypertension and obesity: Mechanisms and implications. J Am Coll Nutr 2001; 20(5 Suppl):428S-435S.

15. Mei C, Tang AM, Zhuxuan F, Wang DD, Newberry SJ. Calcium intake and cardiovascular disease risk: An updated systematic review and meta-analysis. Ann Intern Med 2016; 165:856-866.

16. Heaney RP. Ethnicity, bone status, and the calcium requirement. Nutr Res 2002;22:153-178.

17. Redmond J, Jarjou LM, Zhou B, Prentice A, Schoenmakers I. Ethnic differences in calcium, phosphate and bone metabolism. Proc Nutr Soc 2014;73:340-351.

18. Bryant RJ, Wastney ME, Martin BR, et al. Racial differences in bone turnover and calcium metabolism in adolescent females. J Clin Endocrinol Metab 2003;88:1043-1047.

19. Thomas PA. Racial and ethnic differences in osteoporosis. J Am Acad Orthop Surg 2007;15:S26-S30.

20. Patrelli TS, Dall'Asta A, Gizzo S, et al. Calcium supplementation and prevention of preeclampsia: A meta-analysis. J Matern Fetal Neonatal Med 2012;25:2570-2574.

21. Hofmeyr GJ, Lawrie TA, Atallah ÁN, Torloni MR. Calcium supplementation during pregnancy for preventing hypertensive disorders and related problems. Cochrane Database Syst Rev 2018;10:CD001059.

22. Bischoff-Ferrari HA, Willett WC, Oray EJ, et al. A pooled analysis of vitamin D dose requirements for fracture prevention. N Engl J Med 2012;367:40-49.

23. Moyer VA, U.S. Preventive Services Task Force. Vitamin D and calcium supplementation to prevent fractures in adults: U.S. Preventive Services Task Force recommendation statement. Ann Intern Med 2013;158:691-696.

24. Casanueva E, Viteri FE. Iron and oxidative stress in pregnancy. J Nutr 2003;133:1700S-1708S.

25. Freire AC, Basit AW, Choudhary R, Piong CW, Merchant HA. Does sex matter? The influence of gender on gastrointestinal physiology and drug delivery. Int J Pharm 2011;415:15-28.

26. Office of Dietary Supplements, NIH. Iron fact sheet for health professionals. 2020. Available at: https://ods.od.nih .gov/factsheets/Iron-HealthProfessional Accessed May 11, 2020. 
27. Fernández-Ballart JD. Iron metabolism during pregnancy. Clin Drug Investig 2000;19(Supplement 1):9-19.

28. Siu AL. Screening for iron deficiency anemia and iron supplementation in pregnant women to improve maternal health and birth outcomes: U.S. Preventive Services Task Force Recommendation Statement. Ann Intern Med 2015; 163:529-536.

29. Georgieff MK, Krebs NF, Cusick SE. The benefits and risks of iron supplementation in pregnancy and childhood. Ann Rev Nutr 2019;39:121-146.

30. Fisher AL, Nemeth E. Iron homeostasis during pregnancy. Am J Clin Nutr 2017;106(Supplement 6):1567S-1574S.

31. Harris EL, McLaren CE, Reboussin DM, et al. Serum ferritin and transferrin saturation in Asians and Pacific Islanders. Arch Intern Med 2007;167:722-726.

32. Gordeuk VR, Brannon PM. Ethnic and genetic factors of iron status in women of reproductive age. Am J Clin Nutr 2017;106(Supplement 6):1594S-1599S.

33. Blankson ML, Goldenberg RL, Cutter G, Cliver SP. The relationship between maternal hematocrit and pregnancy outcome: Black-White differences. J Natl Med Assoc 1993; 85:130-134.

34. Khambalia AZ, Aimone A, Nagubandi P, et al. High maternal iron status, dietary iron intake and iron supplement use in pregnancy and risk of gestational diabetes mellitus: A prospective study and systematic review. Diabet Med 2016;33:1211-1221.

35. Zhang C, Rawal S. Dietary iron intake, iron status, and gestational diabetes. Am J Clin Nutr 2017;106(Supplement 6):1672S-1680S.

36. Sunkara A, Raizner A. Supplemental vitamins and minerals for cardiovascular disease prevention and treatment. Methodist Debakey Cardiovasc J 2019;15:179-184.

37. Ingles DP, Cruz Rodriguez JB, Garcia H. Supplemental vitamins and minerals for cardiovascular disease prevention and treatment. Curr Cardiol Rep 2020;22: doi:10.1007/s11886020-1270-1

38. Dragan S, Buleu F, Christodorescu R, et al. Benefits of multiple micronutrient supplementation in heart failure: A comprehensive review. Crit Rev Food Sci Nutr 2019;59:965-981.

39. Yan S, Li M, Ma X, et al. Association of multiple mineral and vitamin B group intake with blood glucose using quantile regression analysis: NHANES 2007-2014. Food Nutr Res 2019;63. DOI:10.29219/fnr.v63.3560.

40. Liu X, Wang B, Li Y, et al. Powerful anticolon tumor effect of targeted gene immunotherapy using folate-modified nanoparticle delivery of CCL19 to activate the immune system. ACS Cent Sci 2019;5:277-289.

41. Wan Ismail WR, Abdul Rahman R, Rahman NAA, Atil A, Nawi AM. The protective effect of maternal folic acid supplementation on childhood cancer: A systematic review and meta-analysis of case-control studies. J Prev Med Public Health 2019;52:205-213.

42. Irwin RE, Pentieva K, Cassidy T, et al. The interplay between DNA methylation, folate and neurocognitive development. Epigenomics 2016;8:863-879.

43. Liu L, Liu S, Wang C, et al. Folate supplementation for methotrexate therapy in patients with rheumatoid arthritis: A systematic review. J Clin Rheumatol 2019;25:197-202.

44. Dixit R, Nettem S, Madan SS, et al. Folate supplementation in people with sickle cell disease. Cochrane Database Syst Rev 2018;3. doi:10.1002/14651858.CD011130.pub3
45. Beik J, Jafariyan M, Montazerabadi A, et al. The benefits of folic acid-modified gold nanoparticles in CT-based molecular imaging: Radiation dose reduction and image contrast enhancement. Artif Cells Nanomed Biotechnol 2018; 46:1993-2001.

46. Naderi N, House JD. Recent developments in folate nutrition. Adv Food Nutr Res 2018;83:195-213.

47. Kim YI. Folate and cancer: A tale of Dr. Jekyll and Mr. Hyde? Am J Clin Nutr 2018;107:139-142.

48. Office of Dietary Supplements (ODS), NIH. Folate fact sheet for health professionals. 2020. Available at: https:// ods.od.nih.gov/factsheets/Folate-HealthProfessional/ Accessed May 11, 2020.

49. U.S. Department of Agriculture, Agricultural Research Service. FoodData Central. 2019. Available at: https://fdc .nal.usda.gov/ Accessed May 11, 2020.

50. Viswanathan M, Treiman KA, Doto JK, Middleton JC, Coker-Schwimmer EJL, Nicholson WK. Folic acid supplementation: An evidence review for the U.S. Preventive Services Task Force. 2017. Report No. 14-05214-EF-1. Available at: https://pubmed.ncbi.nlm.nih.gov/28151610/ Accessed May 11, 2020.

51. Food and Drug Administration. Health claims: Folate and neural tube defects; 21 C.F.R. $\S$ 101.79. 2019. Available at: https://www.accessdata.fda.gov/scripts/cdrh/cfdocs/cfcfr/ CFRSearch.cfm?fr=101.79 Accessed May 11, 2020.

52. Maulik D, van Haandel L, Allsworth J, Chaisanguanthum KS, Yeast JD, Leeder JS. The effect of race and supplementation on maternal and umbilical cord plasma folates. J Matern Fetal Neonatal Med 2019;1-9. DOI:10.1080/14767058.2019 .1677597

53. Mukhtar A, Kramer MR, Oakley Jr GP, Kancherla V. Race and ethnicity and preconception folic acid supplement use among pregnant women in Georgia, PRAMS 2009 to 2011. Birth Defects Res 2017;109:38-48.

54. Lynen R, Taylor TN, Farkouh RA, Candrilli SD, Graham J, Colligs A. The effect of age, race, and folic acid-containing supplements on red blood cell folate levels among United States women of child bearing age. Fertil Sterility 2010; 94(4 Supplement):S93.

55. Williams, J, Mai CT, Mulinare J, et al. Updated estimates of neural tube defects prevented by mandatory folic acid fortification-United States, 1995-2011. MMWR Morb Mortal Wkly Rep 2015;64:1-5.

56. Carmel R. Folic acid. In: Shils M, Shike M, Ross A, Caballero B, Cousins RJ, eds. Modern nutrition in health and disease. 11th ed. Baltimore, MD: Lippincott Williams \& Wilkins, 2005:470-481.

57. Bentley TG, Willett WC, Weinstein MC, Kuntz KM. Population-level changes in folate intake by age, gender, and race/ethnicity after folic acid fortification. Am J Public Health 2006;96:2040-2047.

Address correspondence to:

LaVerne L. Brown, PhD Office of Dietary Supplements

National Institutes of Health

6100 Executive Blvd., $3 B 01$

Bethesda, MD 20892-7517

USA

E-mail: laverne.brown@nih.gov 